\title{
Adicción y sistema cannabinoide endógeno: papel del receptor para cannabinoides CB1 en la fisiología de las neuronas dopaminérgicas mesotelencefálicas
}

\author{
Rodriguez de Fonseca, F; Navarro, M. \\ Departamento de Psicobiología. Facultad de Psicología. Universidad Complutense. \\ Enviar correspondencia a: Prof. Fernando Rodríguez de Fonseca. Departamento de Psicobiología. Facultad de Psicología. Universidad \\ Complutense. Campus de Somosaguas. Madrid. 28223. Pspsc10@sis.ucm.es
}

\begin{abstract}
Resumen
El sistema cannabinoide endogeno es un nuevo sistema de comunicación intercelular compuesto por los receptores para cannabinoides CB-1 y CB-2 y varios transmisores lipidicos, que incluyen a la anandamida y el 2-araquidonilglicerol. Los receptores para cannabinoides CB1 y CB2 son la diana farmacológica de los cannabinoides naturales, los compuestos psicoactivos presentes en la preparaciones de Cannabis sativa. que se consumen como droga ilegal. La investigacion en modelos animales ha constatado que los cannabinoides inducen cambios en los sistemas cerebrales de recompensa, en especial sobre las neuronas dopaminérgicas mesotelencefálicas, equiparables a los que inducen otras drogas como los opiáceos y el etanol. El presente trabajo analiza las evidencias anatómicas, bioquímicas y farmacológicas que apoyan el papel del sistema cannabinoide endógeno en la modulación de la transmisión dopaminérgica. El receptor CB1 no sólo se localiza en las neuronas que expresan receptores para dopamina, sino que se expresa también en células dopaminéricas del mesencéfalo y el hipotálamo. La estimulación de los receptores para dopamina D2 es, además, el estímulo más potente capaz de liberar anandamida que se ha descrito hasta la fecha. La liberación de anandamida bloquearía la hiperactividad comportamental asociada a un exceso de señal dopaminergica. Estos hallazgos permiten esperar que fármacos capaces de interferir con el sistema cannabinoide endógeno puedan ser útiles en la terapéutica de procesos con participación dopaminérgica como la adicción a drogas.
\end{abstract}

Palabras clave: cannabis, sistema cannabinoide, receptores cannabinoides, sistema dopaminérgico, adicción.

\section{Summary}

The endogenous cannabinoid transmission is a new cell signalling system constituted by the cannabinoid CB-1 and CB2 receptors, as well as by several lipid transmitters including anandamide and 2-arachidonoylglycerol. The cannabinoid receptors are the pharmacological targets of the psychoactive constituents of cannabis sativa preparations, commonly used as illegal recreational drugs. Several lines of research using animal models have established that cannabinoids are drugs that modify the activity of the brain reward system, specially the physiology of mesotelencephalic dopaminergic neurones, in a way that resemble the actions of ethanol or the opiates. The present work analyse the anatomical, biochemical and pharmacological evidences that support the role of the endogenous cannabinoid system as a modulator of dopamine transmission in the brain. Cannabinoid CB-1 receptor are present in both, dopamine receptor-containing neurones and mesencephalic and hypothalamic dopaminergic neurones. Moreover, the pharmacological stimulation of striatal dopamine D-2 receptors is the most potent activator of anandamide release known to date. The released anandamide will act as an endogenous break to the hyperactivity associated with a high dopaminergic output. These findings allow us to propose that drugs which interfere with the endogenous cannabinoid system might be useful as a therapy in that problems where the dopamine system intervene as addictions.

Key words: cannabis, cannabinoid system, cannabinoid receptors, dopaminergic system, addiction. 


\section{INTRODUCCION.}

E sistema cannabinoide endógeno cerebral está compuesto por el receptor para cannabinoides, CB-1 (Devane et al., 1988) y los ligandos endógenos anandamida (Devane et al., 1992) y 2-araquidonil glicerol (Mechoulam et al., 1995; Stella et al., 1997). Los efectos agudos y crónicos de los derivados del cannabis sativa se producen por la acción del THC, su constituyente psicoactivo principal, sobre los receptores cerebrales específicos CB-1. Los efectos potencialmente adversos del consumo de cannabis pueden incluir entre otros, a los trastornos de ansiedad (Halikas et al., 1985; Rodríguez de Fonseca et al., 1997), el incremento en el riesgo de inicio de síndromes psicóticos (Andreasson et al., 1987; Núñez-Dominguez y GurpeguiFernández, 1997) y la disminución de la eficacia de los neurolépticos (Knudsen y Vilmar, 1984). Los rasgos psicopatológicos asociados a la exposición aguda y crónica al cannabis indican una estrecha conexión entre los sistemas dopaminérgicos (neuronas liberadoras de dopamina y neuronas que expresan receptores dopaminérgicos) y el sistema cannabinoide endógeno. Las neuronas dopaminérgicas, principalmente las pertenecientes a los sistemas nigroestriatal y mesolímbicocortical, se consideran de especial importancia en los procesos de recompensa y estrés, en la adicción a drogas y en los síntomas positivos de la esquizofrenia (Grace, 1991; Le Moal y Simon, 1991).

Existe un número creciente de trabajos que indican una posible implicación del sistema cannabinoide endógeno en enfermedades relacionadas con el sistema dopaminérgico tales como la adicción (Gardner y Lewinson 1991; Navarro et al., 1995), el estrés (Rodriguez de Fonseca et al., 1994), la psicosis (Andreasson et al 1987; Knudsen y Vilmar, 1984; Núñez-Dominguez y GurpeguiFernández, 1997) o síndromes extrapiramidales tales como la enfermedad de Parkinson o las distonías (Clifford, 1983; Glasset et al., 1997; Rodríguez de Fonseca et al 1994). Sin embargo, todavía necesitamos solventar uno de los desafíos más importantes para la adecuada comprensión del sistema cannabinoide: la explicación del papel fisiológico de un sistema densamente presente en áreas cerebrales de proyección dopaminérgica, con unas características neurobiológicas altamente preservadas a lo largo de la evolución, pero con un bajo tono de actividad como se ha demostrado en estudios funcionales con antagonistas (Howlett, 1995; Gueudet et al., 1995; Navarro et al., 1997). En el presente trabajo trataremos los aspectos bioquímicos, anatómicos y comportamentales de la interacción entre los sistemas dopaminérgico y cannabinoide. Propondremos un modelo con el cual explorar la posible relevancia de estas interacciones para la comprensión y el tratamiento de la adicción, los trastornos neurodegenerativos tales como la enfermedad de Parkinson y ciertos síndromes psiquiátricos tales como la esquizofrenia.

\section{DISTRIBUCIÓN NEUROANATOMICA DE LOS RECEPTORES PARA CANNABINOI- DES CB-1 EN LOS CIRCUITOS DOPAMI- NERGICOS MESOTELENCEFÁLICOS.}

Los receptores CB-1 están presentes en el cerebro de los mamíferos a concentraciones más altas que cualquier otro receptor ligado a proteínas G (Herkenham et al., 1990; Mailleaux y Vanderhaegen, 1992; Matsuda et al 1993). Se expresan en áreas del sistema nervioso central que contribuyen al control del movimiento (caudado-putamen, globus pallidum, núcleo entopeduncular, sustancia negra y cerebelo), cognición y memoria (formación hipocampal, cortex cingulado), procesamiento de emociones y respuestas motivacionales (complejo amigdalar, núcleo accumbens, cortex olfatorio), percepción dolorosa (sustancia gris central, asta dorsal de la médula espinal), e integración neuroendocrina (núcleos paraventricular, arcuato, supraóptico y ventromedial), (Mailleaux y Vanderhaeghen, 1992; Matsuda et al 1993). Del análisis anatómico resulta evidente que el perfil farmacológico de los agonistas CB-1 se corresponde con la distribución de los sitios de unión CB-1 y del 
RNAm del CB-1. Para confirmar este perfil neuroanatómico específico se ha utilizado el SR141716A, antagonista selectivo del receptor CB-1, descrito recientemente (Rinaldi-Carmona et al., 1994) que ha revelado la existencia de un tono endocannabinoide en el hipocampo, sustancia negra y sistema límbico que también se corresponde con la distribución neuroanatómica de los receptores CB1 (Gessa et al., 1997; Gueudet et al., 1995; Navarro et al., 1997: Rodríguez de Fonseca et al., 1997).

Aunque se había descrito la presencia de receptores CB-1 en áreas cerebrales específicas relacionadas con los circuitos dopaminérgicos, tales como los ganglios basales, la amígdala ampliada y el cortex límbico, parecía que no estaban presentes en las neuronas dopaminérgicas cerebrales (Herkenham et al., 1990; Matsuda et al., 1993). Sin embargo estudios más recientes (Ong y Mackie, 1999; Rodríguez de Fonseca et al., datos no publicados) han revelado diferencias específicas de especie en la distribución de los receptores CB-1 en las neuronas dopaminérgicas cerebrales. Observaciones recientes mediante microscopía confocal en ratas de la cepa Wistar (Figura 1) utilizando un anticuerpo policlonal contra el extremo amino-terminal del receptor $\mathrm{CB} 1$, han mostrado que las neuronas dopaminérgicas mesencefálicas expresan una cantidad entre baja y moderada de receptores CB-1, a diferencia de lo que se había descrito previamente en estudios utilizando ratas de la cepa Sprague-Dawley (Herkenham et al ., 1991; Matsuda et al 1993; Tsou et al., 1998). Se han publicado hallazgos similares en el cerebro de primates (Ong y Mackie, 1999), que apoyan la posibilidad de una acción directa de los agonistas del receptor CB-1 en neuronas dopaminérgicas, como se había sugerido en estudios electrofisiológicos previos con antagonistas de los receptores CB-1 (French et al., 1997; Guedet et al., 1995). Sin embargo generalmente se acepta que que los receptores CB-1 se colocalizan con receptores dopaminérgicos en neuronas de áreas de proyección dopaminérgica tales como los ganglios basales y cortex límbico

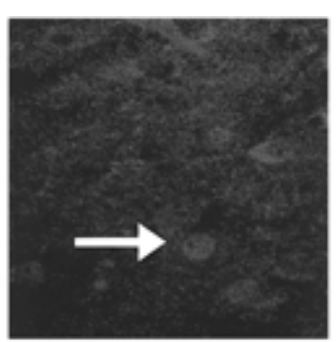

\section{$\mathrm{CB} 1+$}

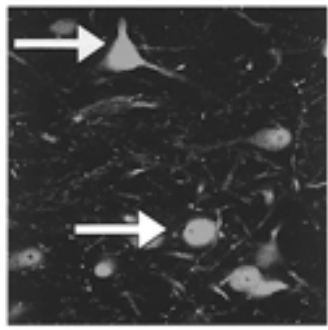

TH +

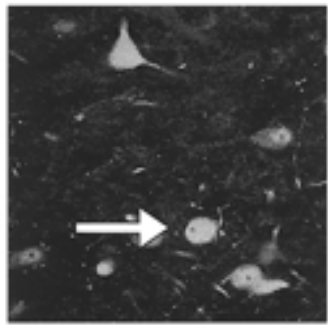

$\mathbf{C B 1}+/ \mathbf{T H}+$

\section{AREA TEGMENTAL VENTRAL}

Figura 1.

Distribución neuroanatómica de los receptores CB1 en el área tegmental ventral del cerebro de la rata Wistar, como se aprecia con doble marcaje inmunocitoquímico y microscopía confocal. La fluorescencia roja corresponde a las células inmunopositivas para tirosina hidroxilasa (TH), mientras que la fluorescencia verde revela la localización de los receptores cannabinoides CB-1. El doble marcaje aparece como una fluorescencia naranja. Los detalles metodológicos se han descrito previamente (Rodriguez de Fonseca et al., 1999). Imagen superior: imagen de células del tegmento ventral que expresan receptor CB-1. Imagen Intermedia: imagen de células del tegmento ventral que expresan tirosina hidroxilasa. Imagen Inferior: doble marcaje para TH y receptor CB-1. La célula marcada con la flecha blanca co-expresa ambos marcadores. La célula marcada con la flecha blanca coexpresa ambos marcadores. La célula marcada con la flecha amarilla sólo expresa TH. 
(Herkenham et al., 1991; Mailleux y Vanderhaeghen, 1993), ya que ambos tipos de receptores están localizados sobre todo en neuronas de proyección, principalmente gabaérgicas, aunque también glutamatérgicas del cortex y del núcleo subtalámico (Rodríguez de Fonseca et al., 1998; SañudoPeña y Walker 1997). En el circuito de los ganglios basales, las neuronas intrínsicas estriatales gabaérgicas espinosas medianas, que reciben aferentes desde las neuronas dopaminérgicas de la sustancia negra pars compacta, coexpresan receptores dopaminérgicos D-1, D-2 y D-3 (Surmeier et al., 1996) y receptores CB-1. Sus terminales axónicos que inervan el globus pallidum, la sustancia negra pars reticulata y el núcleo subtalámico contienen grandes cantidades de receptores CB-1 (Herkenham et al., 1991). La Figura 2, ilustra la posición de los receptores CB-1 en los ganglios basales, en los que participaría en el control de sinapsis relevantes para el procesamiento estriatal de la información compleja procedente del cortex cerebral motor, somatosensorial y límbico.

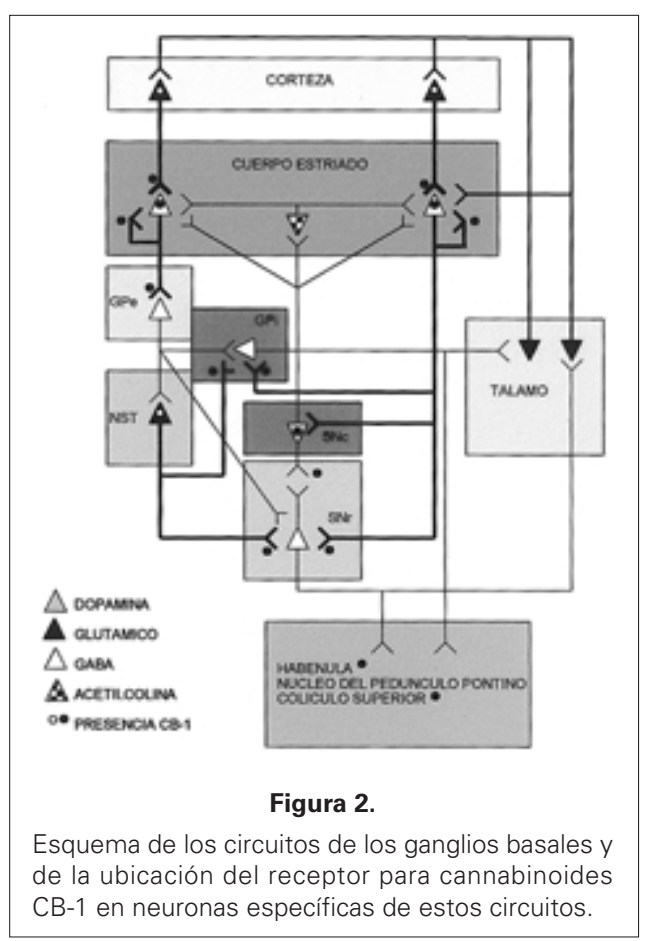

\section{INTERACCIONES SISTEMA ENDOCAN- NABINOIDE-DOPAMINA.}

En las áreas cerebrales descritas anteriormente, relevantes para la mayoría de enfermedades neuropsiquiátricas, la estimulación de los receptores CB-1 podría modular, bien directamente la actividad de las neuronas dopaminégicas (Afectando a la actividad eléctrica, la síntesis, la liberación o la recaptación de dopamina) o bien interferir con la transmisión de la señal dopaminérgica en los receptores dopaminérgicos postsinápticos colocalizados con los receptores CB-1. Distintas aproximaciones experimentales han demostrado ambas posibilidades. La interacción entre receptores de dopamina y receptores CB-1, tiene un apoyo neurobiológico en la estructura similar de los dos tipos de receptores. Ambos tipos pertenecen a la familia de receptores ligados a la proteína G (Matsuda et al., 1991; Howlett, 1995), y ambos también, están acoplados al mismo sistema de transducción de señales, incluyendo el control de la síntesis de AMPc, y la regulación de los canales de $\mathrm{Ca}^{2+}$ y de $\mathrm{K}^{+}$(Hampson et al., 1995; Howlett, 1995; Mackie y Hille, 1992).

\section{Interacciones dopamina-cannabinoide en el sistema nigroestriatal.}

El papel del sistema cannabinoide endógeno parece ser diferente según el nivel de los diferentes circuitos dopaminérgicos. La mayoría de los efectos observados en el sistema nigroestriatal indican una regulación indirecta de la actividad dopaminérgica, mediante la estimulación de los receptores CB-1 que expresan las neuronas gabaérgicas de los núcleos eferentes de los ganglios basales (Maneuf et al., 1996; Navarro et al., 1993b; Pertwee y Greetree, 1998). No se han descrito alteraciónes marcadas del turnover de dopamina a nivel estriatal tras la exposición aguda a THC, ni en Sprague-Dawley (Rodríguez de Fonseca et al., 1992) ni en ratas Wistar (Navarro et al., 1993). Sin embargo, se encontró que la administración aguda de agonistas del recetor cannabinoide produ- 
cía un pequeño incremento de la actividad espontánea de las neuronas dopaminérgicas nigroestriatales medidas bien por registro extracelular (French et al., 1997), o por voltametria in vivo en estriado ( $\mathrm{Ng}$ Cheon et al., 1988). La naturaleza directa o indirecta de estos efectos permanece sin determinar de forma concluyente. Sin embargo, un estudio reciente ha clarificado en parte la relación entre el sistema cannabinoide endógeno y la transmisión dopaminérgica (Guiuffrida et al., 1999). En este estudio, con la técnica de microdiálisis in vivo hemos demostrado que los niveles extracelulares de anandamida en estriado dorsal sufren un gran incremento tras la estimulación de la familia de receptores dopaminérgicos D-2 (p.e. tras la infusión de quinpirole mediante diálisis inversa), pero no tras la estimulación receptores dopaminérgicos D-1 (p.e. tras la infusión con el agonista D-1 SKF 38393). La anandamida liberada tras estimulación del receptor D-2 puede ser útil para limitar el aumento de conducta motora producida por la dopamina en el estriado. Como se muestra en las figuras 3 y 4 el pretratamiento con el antagonista CB-1 SR141716A potencia la estimulación de la conducta motora producida por la administración sistémica de quinpirole. Este efecto no se observó cuando el efecto facilitatorio sobre el movimiento fue inducido con un agonista D-1, el SKF 81297. Otros laboratorios han descrito interacciones farmacológicas entre receptores dopaminérgicos D-2 y receptores cannabinoides $\mathrm{CB}-1$ que apoyan esta hipótesis. El grupo de J. Michael Walker ha descrito que la administración regional de agonistas de la familia D-2 reduce la respuesta conductual producida por la inyección de agonistas CB-1.

Aunque el aumento de la liberación de anandamida parece ser dependiente de la estimulación de los receptores D-2, los receptores dopaminérgicos D-1 juegan también un papel importante en esta interacción cannabinoide-dopamina. Así, un estudio previo (Mailleux y Vanderhaeghen, 1993) demostró que el bloqueo crónico de los receptores D-1, que produce una hiperactividad compensatoria en células nigroestriatales dopaminérgicas, provoca un gran aumento en la expresión de RNAm del receptor CB-1 en el estriado dorsal. Por otro lado, la estimulación repetida de los receptores dopaminérgicos D-1 y D-2 y la consiguiente desensibilización de ambos sistemas receptoriales da lugar a una potenciación de la acinesia y de la catalepsia inducidas por al agonista cannabinoide HU-210 (Rodríguez de Fonseca et al., 1994b). El efecto opuesto se observa cuando se sensibilizan los receptores D1 mediante la administración repetida del neuroléptico SCH 23390, un bloqueante selectivo del receptor D1 (Rodrìguez de Fonseca et al., 1998). Estos datos sugieren la existencia de diferencias en las interacciones entre los sistemas dopminérgico y cannabinoide considerando los distintos receptores

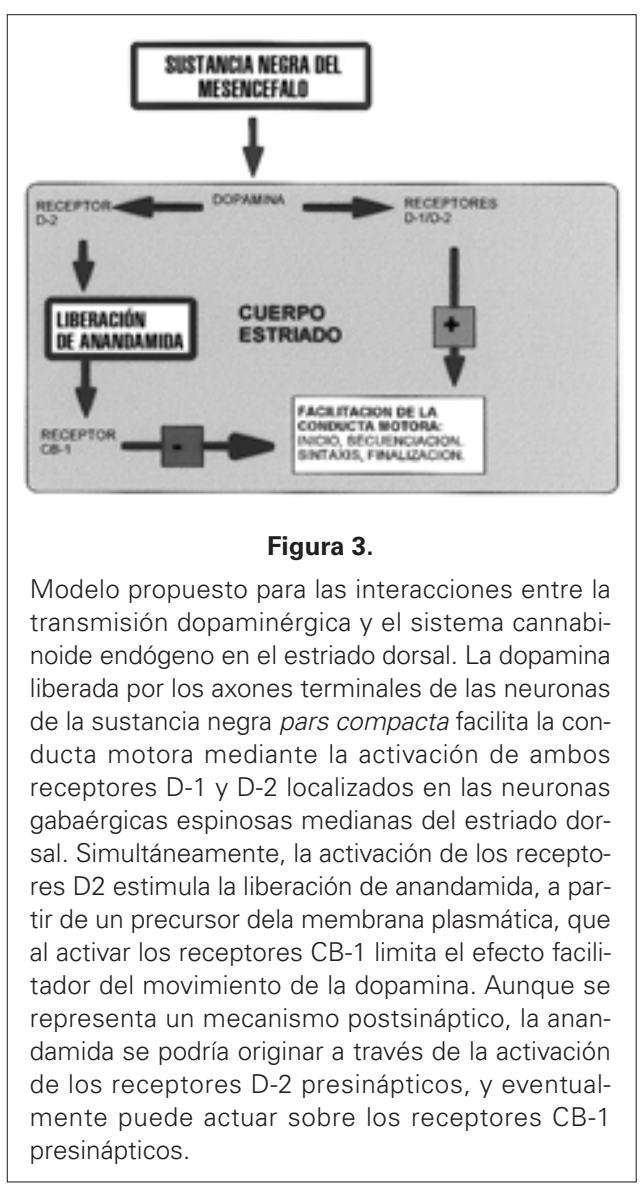




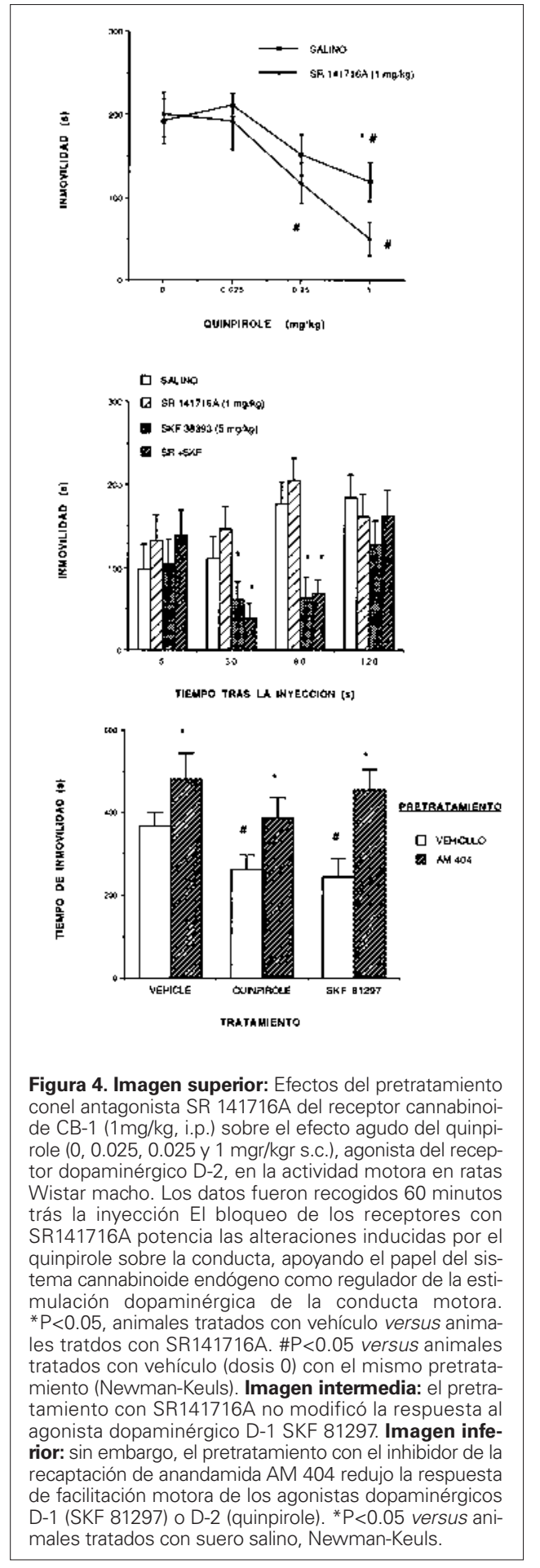

involucrados. La figura 2 representa una hipótesis de trabajo actual sobre la interacción entre receptores cannabinoides y dopaminérgicos en estriado. Todavía está por determinar si estos efectos farmacológicos son selectivos del estriado dorsal o aparecen en otras áreas cerebrales. Se ha propuesto la existencia de diferencias regionales en el papel de los receptores CB-1 sobre el control de la actividad de los ganglios basales desde el mesencéfalo debido a la gran densidad de receptores dopaminérgicos en la sustancia negra (Glass et al., 1997a). Así, se ha descrito que los cannabinoides activan las neuronas de la sustancia negra pars reticulata, probablemente inhibiendo la liberación de GABA desde las proyecciones estriatonígricas mediante la estimulación de los receptores CB-1 presinápticos (Tersigni y Rosemberg, 1996). Este bloqueo de la liberación de GABA puede ser el responsable del aumento de la actividad de las neuronas dopaminérgicas nigroestriatales observado in vivo tras la exposición aguda a cannabinoides, ya que están bajo la influencia de la via striatonígrica (French et al., 1997; Gueudet et al., 1995; Ng Cheong el al., 1988). Una fuente adicional indirecta de aferentes reguladores sobre la sustancia negra, cuya actividad puede ser modulada por la estimulación CB-1, proviene del núcleo subtalámico (Sañudo-Peña y Walker, 1997). Por otro lado, los receptores cannabinoides en estriado parece que están acoplados negativamente a la liberación de dopamina estimulada por $\mathrm{K}^{+}$ (Navarro et al., 1993b) o evocada eléctricamente (Cadogan et al., 1997) como se demostró por estudios in vitro. Un balance entre la actividad de las influencias convergentes en la sustancia negra y las actividades reguladoras locales establecerán la naturaleza de las acciones de los agonistas CB-1 sobre la liberación de dopamina en estriado. Manipulaciones farmacológicas de la sensibilidad aguda a cannabinoides mediante la administración concurrente de drogas que actúan sobre los receptores GABA-A y GABA-B (Pertwee y Greentree, 1988; Romero et al., 1995) apoyan la implicación de las neuronas GABA en la mediación de los efectos cannabinoides. Esta contribución se deduce tam- 
bién del hecho de que el agonista del receptor CB-1, induce cambios en los receptores dopaminérgicos estriatales (Navarro et al., 1993b; Rodríguez de Fonseca et al., 1992). Estos efectos se pueden poner de manifiesto comportamentalmente 24 horas después de una administración única de THC. (Figura 5).

\section{Interacción dopamina-cannabinoide en los circuitos mesolímbicos.}

El sistema dopaminérgico mesocorticolímbico es más sensible que la vía nigroestriatal a la administración aguda de cannabinoides.

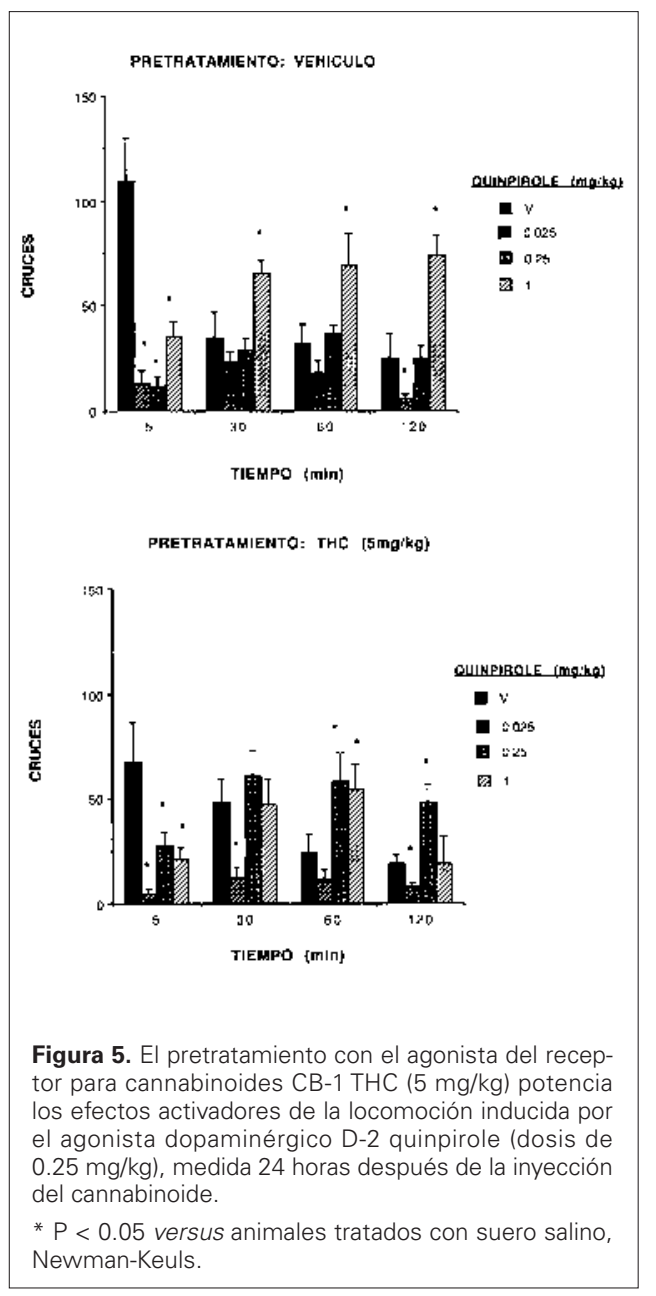

Las primeras descripciones de las acciones del THC en el cerebro indicaban una estimulación de la actividad dopaminérgica mesocorticolímbica (Bowers y Hoffman, 1986; Chen et al., 1990). Registros extracelulares directos mostraron que la administración sistémica de agonistas CB-1 incrementaban la actividad de neuronas dopaminérgicas del área tegmental ventral (French et al., 1997) asociadas a un aumento de la liberación de dopamina en las terminales mesolímbicas (tabla 1; Gardner et al., 1988; Navarro et al., 1993a; Tanda et al., 1997). Según estudios preliminares del grupo de Gardner (Gardner y Vorel, 1998), la liberación de dopamina inducida por cannabinoides es aditiva a la producida por el bloqueo de receptores dopaminérgicos D-2, lo que sugiere que el receptor CB-1 podría oponerse a la acción de los autorreceptores que controlan la liberación de esta monoamina. Sin embargo, está por determinar de forma concluyente si este efecto es producido por la estimulación directa de los receptores CB-1 presentes en células dopaminérgicas, por estimulación transináptica.o por activación del eje hipotálamo-hipófiso-adrenal. Los trabajos realizados hasta la fecha son contradictorios a este respecto, y son necesarias investigaciones adicionales para establecer concluyentemente el papel de los receptores de dopamina D-1 y D-2 en la respuesta de las neuronas dopaminérgicas mesocorticolímbicas a la exposición aguda a cannabinoides. Por ejemplo, el bloqueo de los receptores CB-1 con el antagonista selectivo SR141617A no afecta la actividad del área tegmental ventral (Gueudet et al., 1995), mientras que los antagonistas de los receptores opioides-m (naloxona, naloxonazina) bloquean el aumento de la liberación de dopamina inducida por la administración de agonistas CB-1, actuando ambos probablemente sobre las neuronas del área tegmental ventral (Tanda et al 1997) y en sus extremos terminales en el núcleo accumbens (Chen et al., 1990; Gardner y Lowinson, 1991). Una hipótesis adicional interesante es que la activación mesolímbica inducida por los agonistas CB-1 sea glucocorticoide-dependiente. Los cannabinoides son estresores químicos que activan el eje hipofi- 
so-adrenal mediante liberación de la ACTH (Martín Calderón et al., 1998). También inducen respuestas de ansiedad (Rodriguez de Fonseca et al 1997). El estrés agudo se asocia con una activación rápida de la vía mesolímbica que puede estar mediada por los receptores de glucocorticoides presentes en neuronas dopaminérgicas mesolímbicas (Kalivas y Stewart, 1991; Piazza et al., 1996). Está por determinar si la activación mesolímbica depende de la activación del eje hipofiso-adrenal, pero su demostración puede servir de soporte a las observaciones clínicas sobre el papel del consumo de cannabis como factor de vulnerabilidad en el desencadenamiento de un brote psicótico o en la drogadicción (Andreasson et al., 1987; Navarro et al., 1995; Rodríguez de Fonseca et al., 1997). La reciente descripción de la aparición de sensibilización a psicoestimulantes en animales tratados crónicamente con el agonista CB-1 THC apoya esta hipótesis, y refuerza el papel relevante del sistema cannabinoide endógeno en los fenómenos que conducen al establecimiento de la conducta adictiva. En cualquier caso, la administración crónica de agonistas cannabinoides induce cambios importantes en la funcionalidad de las neuronas dopaminérgicas mesolímbicas y mesocorticales. Diana y colaboradores han demostrado que estas neuronas sufren cambios adaptativos a resultas de la administración crónica de cannabinoides que se asemejan a los descritos tras la administración crónica de etanol u opiáceos. Estos cambios se manifiestan como una reducción en la actividad eléctrica espontánea de estas neuronas durante la instauración de abstinencia a cannabinoides inducida farmacológicamente (mediante la inyección del antagonista SR 141716 A) o de manera espontànea (cese de administración de agonistas (B-1). Este declinar en la actividad espontànea mesocorticolímbica se asocia al estado afectivo negativo, disfórico, que acompaña al cese de la toma de drogas de abuso, y que se considera un factor importante en el mantenimiento del consumo de la droga y en las recaídas.

\section{PAPEL DE LAS INTERACCIONES CAN- NABINOIDE EN LA PATOGENIA Y TRA- TAMIENTO DE ENFERMEDADES NEU- ROPSIQUIÀTRICAS.}

Como hemos descrito anteriormente, los cannabinoides endógenos son mediadores locales liberados para regular el procesado de la información en las principales conexiones de los núcleos de los ganglios basales, y posiblemente en las vías del refuerzo. La posible actividad constitutiva de los receptores CB-1 y su acoplamiento bidireccional a la adenilato ciclasa sugiere que esta función reguladora afecta a procesos de naturaleza opuesta dentro del estriado, indicando un posible papel para este sistema como un potencial regulador de la homeostasis local (Glass y Felder, 1997b; Maneuf y Brotchie, 1997; Rodríguez de Fonseca et al., 1998). La inducción de un bloqueo funcional del proceso de recaptación de neurotransmidores derivado de la estimulación CB-1 puede afectar a neurotransmisores de naturaleza opuesta, tales como glutamato y GABA, apoyando nuevamente el papel amortiguador de la señal cannabinoide endógena en el estriado. Queda pendiente el identificar las posibles variaciones regionales en estos mecanismos, así como las condiciones patológicas en las cuales una clara contribución del sistema cannabinoide endógeno puede contribuir a mejorar nuestro conocimiento de ciertos trastornos neurológicos. Los hallazgos de investigación tratados en este manuscrito sugieren que el sistema cannabinoide endógeno puede servir de objetivo para el desarrollo de nuevas estrategias para el tratamiento de procesos relacionados con la transmisión dopaminérgica. En el campo de la adicción a drogas, la utilización de agonistas parciales débiles del receptor CB-1 podría, teóricamente, ayudar a reducir el consumo de psicoestimulantes, opiáceos o cannabinoides. Los antagonistas del receptor CB-1 podrían ayudar a reducir el deseo compulsivo de toma de drogas durante la abstinencia o tras la misma, especialmente en individuos con alta tasa de consumo. Esta aproximación ya ha sido ensayada con éxito en la dependencia a opiáceos o a etanol (Ver Navarro et al., este 
volumen o Rodríguez de Fonseca et al., 1999). De entre los trastornos del movimiento, la enfermedad de Parkinson, las discinesias tardías y las distonías inducidas por neurolépticos son firmes candidatos a beneficiarse de la terapia basada en el sistema cannabinoide endógeno, junto al síndrome de Gilles de la Tourette y la corea de Huntington (Rodríguez de Fonseca et al., 1998). Como una potencial utilidad práctica del modelo podemos proponer los agonistas cannabinoides para reducir los efectos indeseados de la L-dopa o agonistas dopaminérgicos en la enfermedad de Parkinson o en las discinesias tardías asociadas al uso de neurolépticos, así como antagonistas cannabinoides para reducir la dosis efectiva de L-DOPA o agonistas dopaminérgicos necesarios para aliviar la acinesia característica de dicha enfermedad (Maneuf et al., 1997; Rodríguez de Fonseca et al., 1998).

Además de los trastornos motores, también se podrían beneficiar distintas alteraciones neuropsiquiátricas de la disponibilidad de nuevos compuestos que actuasen sobre los receptores cannábicos, tales como los nuevos antagonistas selectivos, así como del esclarecimiento de nuevos mecanismos moleculares y de la identificación de nuevas dianas farmacológicas, tales como el recientemente descrito transportador de anandamida (Beltramo et al., 1997). Este último hallazgo ha abierto múltiples posibilidades. Un ejemplo cardinal pueden ser los síntomas agudos de la esquizofrenia, que actualmente son atribuidos, entre otros mecanismos, a un estado hiperdopaminérgico. Si la liberación de anandamida también se dispara en áreas corticales tras la activación de los receptores D-2, un aumento de la biodisponibilidad de anandamida por medio del bloqueo de la recaptación de anandamida o de la hidrólisis pueden actuar como terapia antipsicótica. Estudios experimentales (Beltramo et al., 2000) en animales de laboratorio han demostrado esta hipótesis, así como la de la normalización de la hiperactividad infantil en un modelo de ratas genéticamente hiperactivas. Del mismo modo, la administración crónica de un antagonista del receptor CB-1 podría servir para reducir la sintomatología negativa asociada a la esquizofrenia, si ésta se debiese a la reducción de la señal dopaminèrgica en las áreas de proyección corticolímbicas.

Por último, el hecho de que la anandamida sea un agonista parcial puede evitar los efectos colaterales indeseables producidos por los agonistas completos como la catalepsia o las reacciones de estrés agudas Aunque de la rata se ha descrito que el tratamiento crónico con THC puede inducir pérdida neuronal en el hipocampo, de naturaleza glucocorticoidedependiente (Landfield et al., 1988), también se han descrito acciones neuroprotectoras derivadas de la estimulación del receptor cannabinoide CB-1 in vitro e in vivo (Nagayama et al., 1999). Estos hallazgos indican que un uso racional de estas drogas dirigidas al receptor CB-1, puede proteger a las neuronas de diferentes tipos de agresiones. Continuando con este razonamiento, compuestos que activasen el receptor CB-1 o incrementasen la liberación de endocannabinoides podrían, además, tener propiedades preventivas del deterioro al actuar como neuroprotectores (Por ejemplo, al disminuir la liberación de glutámico o disminuir la neurotoxicidad mediada por los receptores NMDA).

\section{AGRADECIMIENTOS.}

Este trabajo ha sido financiado por los Proyectos de la Comunidad de Madrid (08.5/0013/98 y 05/0024/99), por el Hospital Psiquiátrico de Ciempozuelos-Orden de San Juan de Dios, LA DGICYT, proyecto PM 96/0047 y La Delegación del Gobierno para el Plan Nacional Sobre Drogas. Los autores agradecen especialmente la colaboración de los Doctores Luis Miguel García Segura y Miguel Angel Gorriti, así como la asistencia técnica de Concepción Bailón del Instituto Cajal, CSIC, Madrid.

\section{REFERENCIAS BIBLIOGRAFICAS.}

Anderson LA, Anderson JJ, Chase TN and Walters JR (1995) The cannabinoid agonist WIN 55,212- 
2 and CP 55,940 attenuate rotational behaviour induced by a dopamine D1 but not a D2 agonist in rats with unilateral lesions of the nigrostriatal pathway. Brain Res., 691, 106-114.

Andreàsson $S$, Allebeck $P$, Engström $A$, Rydberg $V$ (1987) Cannabis and Schizophrenia: a longitudinal study of Swedish conscripts. Lancet, ii, 1483-1486.

Beltramo M, Rodríguez de Fonseca F, Navarro $M$, Calignano A, Gorriti MA, Sadile AG, Giuffrida A, Piomelli D. (2000) Reversal of dopamine D-2 receptor-mediated responses by an anandamide transport inhibitor. J. Neuroscience (En prensa)

Beltramo M, Stella N, Calignano A, Lin SY, Makriyannis A and Piomelli D (1997) Functional role of high-affinity anandamide transport as revealed by selective inhibition. Science, 277, 10941097.

Cadogan AK, Alexander SPH, Boyd EA and Kendal DA (1997) Influence of cannabinoids on electrically evoked dopamine release and cAMP generation in the rat striatum. J. Neurochem., 69, 1131-1137

Chen J, Paredes W, Li J, Smith D, Lowinson J and Gardner EL (1990) Delta-9-tetrahydrocannabinol produces naloxone-blockable enhancement of presynaptic basal dopamine efflux in nucleus accumbens of conscious, freelymoving rats as measured by intracerebral microdialysis. Psychopharmacol., 102, 156-162.

Clifford DB (1983) Tetrahydrocannabinol for tremor in multiple sclerosis. Ann. Neurol., 13, 669-671.

Devane WA, Dysarz FA III, Johnson MR, Melvin LS and Howlett AC (1988) Determination and characterization of a cannabinoid receptor in rat brain. Mol. Pharmacol., 34, 605-613.

Devane WA, Hanus L, Breuer A, Pertwee RG, Stevenson LA, Griffin G, Gibson D, Mandelbaum A, Etinger A and Mechoulam R (1992) Isolation and structure of a brain constituent that binds to the cannabinoid receptor. Nature, 258, 19461949.

Dewey WL (1986) Cannabinoid Pharmacology. Pharmacol. Rev. , 38, 151-178.

Diana M, Melis M, Gessa GL (1998) Cannabinoids activate mesolimbic dopamine neurons by an action on cannabinoid CB1 receptors. Eur. J. Pharmacol. 341, 39-44.

Diana M, Melis M, Muntoni AL, Gessa GL. (1998) Mesolimbic dopaminergic decline after canna- binoid withdrawal. Proc Natl. Acad. Sci. USA, 95, 10269-10273

Diana M, Muntoni AL, Pistis M, Melis M, Gessa GL. (1999) Lasting reduction in mesolimbic dopamine neuronal activity after morphine withdrawal. Eur. J. Neurosci., 11, 1037-1041.

French ED, Dillon K and Wu X (1997) Cannabinoids excite dopamine neurons in the ventral tegmentum and substantia nigra. NeuroReport, 8 , 649-652.

Gaoni Y and Mechoulam R (1964) Isolation, structure and partial synthesis on an active constituent of hashish. J Am. Chem. Soc. , 86, 16461654.

Gardner EL, Paredes W, Smith D, Donner A, Milling C, Cohen D and Morrison D (1988) Facilitation of brain stimulation reward by $\Delta^{9}$-tetrahydrocannabinol. Psychopharmacol., 96, 142-144.

Gardner EL and Vorel, RH (1998) Cannabinoid transmission and reward-related events. Neurobiology of Disease. 5, 502-533.

Gessa, GL, Melis, M, Muntoni AL, Diana M (1998) Cannabinoids activate mesolimbic dopamine neurons by an action on cannabinoid CB1 receptors. Eur. J. Pharmacol. 341, 39-44.

Giuffrida, A., Parsons, L.H., Kehrr, A., Rodríguez de Fonseca, F., Navarro, M., Piomelli, D.(1999) Dopamine activation of endogenous cannabinoid signalling in dorsal striatum. Nature Neuroscience 2, 358-363.

Glass M, Brotchie JM and MAneuf YP (1997a) Modulation of neurotransmission by cannabinoids in the basal ganglia. Eur. J. Neurosci., 9, 199-203.

Glass M and Felder C (1997b) Concurrent stimulation of cannabinoid CB1 and dopamine D2 receptors augments CAMP accumulation in striatal neurons: evidence for a Gs linkage to the CB1 receptor. J. Neurosci., 17, 5327-5333.

Gorriti, M.A., Rodriguez de Fonseca, F.A., Navarro, M., Palomo, M.(1999) Chronic treatment with (-)- delta-9-tetrahydrocannabinol results in sensitization to the psychomotor effects of amphetamine. Eur. J. Pharmacol. 365, 133142.

Grace AA (1991) Phasic versus tonic dopamine release and the modulation of dopamine system responsivity: a hypothesis for the etiology of schizophrenia. Neuroscience, 41, 1-24.

Gueudet C, Santucci V, Rinaldi-Carmona M, Soubrié $P$ and Le Fur G (1995) The CB1 cannabinoid 
receptor antagonist SR 141716A affects A9 dopamine neuronal activity in the rat. NeuroReport , 6, 1293-1297.

Halikas JA, Weller RA, Mouse CL and Hoffman RA (1985) A longitudinal study of marijuana effects. Int. J. Addiction, 20, 701-711.

Hampson RE, Evans GJO, Mu J, Zhuang S, King VC, Childers SR and Deadwyler SA (1995) Role of cyclic AMP dependent protein kinase in cannabinoid receptor modulation of potassium "Acurrent" in cultured hippocampal neurons. Life Sci., 56, 2081-2087

Herkenham M, Lynn AB, de Costa BR and Richfield EK (1991) Neuronal localization of cannabinoid receptors in the basal ganglia of the rat. Brain Res, 547, 267-274.

Herkenham M, Lynn AB, Little MD, Johnson MR, Melvin LS, de Costa BR and Rice (1990) Cannabinoid receptor localization in brain. Proc. Natl. Acad. Sci. USA., 87, 1932-1936.

Howlett AC (1995) Pharmacology of cannabinoid receptors. Ann. Rev. Pharmacol. Toxicol. 35, 607-634.

Knudsen P and Vilmar P (1984) Cannabis and neuroleptics agents in schizophrenia. Acta Psychiatr. Scand., 69, 162-174.

Landfield PW, Cadwallader LB and Vinsant S (1988) Quantitative changes in hippocampal structure following chronic exposure to $\Delta^{9}$-tetrahydrocannabinol: possible mediation by glucocorticoid systems. Brain . Res., 443, 47-62.

Le Moal M and Simon H (1991) Mesocorticolimbic dopaminergic network: functional and regulatory roles. Physiol. Rev., 71, 155-234.

Leweke, FM, Giuffrida, A, Wurster, U, Emrich, HM and Piomelli, D (1999) Elevated endogenous cannabinoids in schizophrenia. NeurReport 10, 1665-1669.

Mackie K, and Hille B (1992) Cannabinoids inhibit Ntype calcium channels in neuroblastoma-glioma cells. Proc. Natl. Acad. Sci. USA, 89, 3825-3829.

Mailleux P and Vanderhaeghen JJ (1992) Distribution of neuronal cannabinoid receptor in the adult rat brain: a comparative receptor binding radioautoagraphy and in situ hybridization histochemistry. Neuroscience, 48, 655-688.

Mailleux P and Vanderhaeghen JJ (1993) Dopaminergic regulation of cannabinoid receptor mRNA levels in the rat caudate-putamen: An in situ hybridization study. J. Neurochem., 61, 1705-1712.
Maneuf YP and Brotchie JM (1997) Paradoxical action of the cannabinod WIN 55,212-2 in stimulated and basal cyclic AMP accumulation in rat globus pallidus slices. Br. J. Pharmacol., 120, 1397-1398.

Maneuf YP, Crossman AR and Brotchie JM (1996) Modulation of GABAergic transmission in the globus pallidus by the synthetic canabinoid WIN 55,212-2. Synapse, 22, 383-385.

Maneuf YP, Crossman AR and Brotchie JM (1997) The cannabinoid receptor agonist WIN 55,2122 reduces $D 2$, but not D1, dopamine receptormediated alleviation of akinesia in the reserpine-treated rat model of Parkinson's disease. Exp. Neurol., 148, 265-270

Martín-Calderón JL, Muñoz RM, Villanúa, del Arco I, Moren JL, Rodríguez de Fonseca F and Navarro M (1998) Characterization of the acute endocrine actions of HU-210, a potent synthetic cannabinoid in rats. Eur. J. Pharmacol. 344, 77-86.

Matsuda LA, Bonner TI and Lolait SJ (1993) Localization of Cannabinoid Receptor mRNA in rat brain. J . Comp. Neurol., 327, 535-550

Matsuda LA, Lolait SJ, Brownstein MJ, Young AC and Bonner TI (1990) Structure of a brain cannabinoid receptor and functional expression of the cloned cDNA. Nature, 346, 561-564.

Mechoulam R, Ben-Shabat S, Hanus L, Ligumsky M, Kaminski NE, Schatz AR, Gopher A, Almog S, Martin BR, Comton DR, Pertwee RG, Griffin G, Bayewitch M, Barg J and Vogel Z (1995) Identification of an endogenous 2-monoglyceride present in canine gut that binds to cannabinoid receptor. Biochem. Pharmacol., 50, 83-90.

Nagayama, T., Sinor, A.D., Simon, R.P., Cheen, J., Graham, S.H., Jin, K., Greenberg, D.A. (1999) Cannabinoids and neuroprotection in global and focal cerebral ischemia and in neuronal cultures. J.Neurosci. 19, 2987-95.

Navarro, M, Cowen, J, Carrera, MRA, del Arco, I., Villanúa, MA, Martin Y, Roberts, AJ, Koob, GF and Rodriguez de Fonseca, F (1998) CB1 cannabinoid receptor antagonist-induced opiate withdrawal in morphine-dependent rats. NeuroReport, 9, 3397-3402.

Navarro M, Fernandez-Ruiz JJ, de Miguel R, Hernandez ML, Cebeira M and Ramos JA (1993) Motor disturbances induced by an acute dose of delta9-tetrahydrocannabinol: possible involvement of nigrostriatal dopaminergic alterations. Pharmacol. Biochem. Behav., 45, 291-298. 
Navarro M, Hernández E, Muñoz RM, Del Arco I, Villanúa MA, Carrera MRA and Rodríguez de Fonseca F (1997) Acute administration of the CB1 receptor antagonist SR 141716A induces anxiety-like responses in the rat. NeuroReport, 8, 491-496.

Ng Cheong Ton JM, Gerhardt SA, Freidman M, Etgen A, Rose GM, Sharlegg NS and Gardner EL (1988) The effect of delta-9-tetrahydrocannabinol on potassium-evoked release of dopamine in the rat caudate nucleus: an in vivo electrochemical and in vivo microdialysis study. Brain Res., 451, 59-68.

Nuñez-Domínguez LA and Gurpegui-Fernández de Legaria M (1997) Cannabis psychosis: a five year follow-up study. International Meeting on Interactive Monoaminergic Brain Disorders. October 8-12, 1997, P3.5, pp 76.

Ong, W.Y. and Mackie, K. (1999) A light and electron microscopic study of the CB1 cannabinoid receptor in primate brain. Neuroscience 92 1177-1191.

Pertwee RG and Greentree SG (1988) Delta-9tetrahydrocannabinol-induced catalepsy in mice is enhanced by pretreatment with flurazepam or chlordiazepoxide. Neuropharmacol., 27, 485-491.

Piazza PV and Le Moal M (1996b) Pathophysiological basis of vulnerability to drug abuse: role of an interaction between stress, glucocorticoids and dopaminergic neurons. Ann. Rev. Pharmacol. Toxicol., 36, 359-378.

Rinaldi-Carmona M, Barth F, Héaulme M, Shire D, Calandra B, Congy C, Martinez S, Maruani J, Néliat G, Caput D, Ferrara P, Soubrié, Breliére JC and Le Fur G (1994) SR 141716A a potent and selective antagonist of the brain cannabinoid receptor. FEBS Lett., 350, 240-244.

Rodríguez de Fonseca F, Carrera MRA, Navarro M, Koob GF and Weiss F (1997) Activation of corticotropin-releasing factor in the limbic system during cannabinoid withdrawal. Science, 276, 2050-2054.

Rodríguez de Fonseca F, Fernandez-Ruiz JJ, Murphy LL, Cebeira M, Steger RW, Bartke A and Ramos A (1992) Acute effects of $\Delta^{9}$ tetrahydrocannabinol on dopaminergic activity in several rat brain areas. Pharmacol. Biochem. Behav. 42, 269-275.

Rodríguez de Fonseca F, Gorriti MA, FernándezRuiz JJ, Palomo T and Ramos JA (1994a) Down regulation of rat brain cannabinoid binding sites after chronic $\Delta^{9}$-tetrahydrocannabinol treatment. Pharmacol. Biochem. Behav. , 47, 33-40.

Rodríguez de Fonseca, F.A., Martín-Calderón, J.L., Del Arco, I., Gorriti, M.A.,Navarro, M.(1998) Role of the endogenous cannabinoid system in the regulation of motor activity. Neurobiology of Disease 5, 483-501.

Rodríguez de Fonseca F, Martín-Calderón JL, Mechoulam R and Navarro M (1994b) Repeated stimulation of D-1 dopamine receptors enhances (-)-11-hydroxy-?8-tetrahydrocannabinol-dimethylheptyl-induced catalepsy in male rats. NeuroReport, 5, 761-765.

Rodríguez de Fonseca FA, Roberts AJ, Bilbao A, Koob GF, Navarro M. (2000) Cannabinoid receptor antagonist SR 141716 A decreases operant ethanol self-administration in rats exposed to ethanol-vapor chambers. Acta. Pharmacol. Sinica, 20, 1109-1114.

Rodríguez de Fonseca, F.; Wenger, T., Navarro, M.; Murphy, L.L.(1999) Effects of THC on VIP-induced prolactin secretion in pituitary cultures: evidence for the presence of functional cannabinoid receptors in pituitary cells. Brain Res. 841, 114-122.

Romero J, García-Palomero E, Fernández-Ruiz JJ, and Ramos JA (1995) Involvement of GABA-B receptors in the motor inhibition produced by agonists of brain cannabinoid receptors. Behav. Pharmacol., 7, 299-302.

Sañudo-Peña MC, Patrick SL, Patrick RL and Walker JM (1996) Effects of intranigral cannabinoids on rotational behavior in rats: interactions with the dopaminergic system. Neurosci. Lett., 206, 21-24.

Sañudo-Peña MC, and Walker JM (1997) Role of the subthalamic nucleus in cannabinoid actions in the substantia nigra of the rat.J. Neurophysiol., 77, 1635-1638.

Sañudo-Peña, M.C. and Walker, J.M. (1998). Effects of intrapallidal cannabinoids on rotational behavior in rats. Interaction with the dopaminergic system. Synapse 28, 2-32.

Sim, L.J., Selley, D.E., Dworkin, S.I. and Childers,S.R.(1996) Effects of chronic morphine administration on mu opioid receptor-stimulated [35S]GTPgS autoradiography in rat brain. J. Neurosci., 16, 2684-2692 .

Steiner, H., Bonner, T.I., Zimmer, A.M., Kitai, S.T. and Zimmer, A. (1999) Altered gene expression in striatal projection neurons in CB1 cannabi- 
noid receptor knockout mice. Proc. Natl. Acad. Sci. 96, 5786-5790.

Stella N, Schweitzer P and Pomelli D (1997) A second endogenous cannabinoid that modulates long-term potentiation. Nature, 388, 773777.

Surmeier DJ, Song WJ and Yan Z (1996) Coordinated expression of dopamine receptors in neostriatal medium spiny neurons. J. Neurosci. , 16, 6579-6591.
Tanda, G, Pontieri, FE, Di Chiara, G (1997) Cannabinoid and heroin activation of mesolimbic dopaminetransmission by a common mu1 opioid receptor mechanism. Science 76, 2048-2050.

Tersigni T and Rosemberg HC (1996) Local pressure application of cannabinoid agonists increases spontaneous activity of rat substantia nigra pars reticulata neurons without affecting response to iontophoretically-applied GABA. Brain Res., 733, 184-192

Tsou, K., Sañudo-Peña, M.C., Mackie, K., Walker, J.M. (1998) Immunohystochemical distribution 